\title{
NOTCH2 signaling confers immature morphology and aggressiveness in human hepatocellular carcinoma cells
}

\author{
YOSHIHIRO HAYASHI, MAKOTO OSANAI and GANG-HONG LEE
}

Department of Pathology, Kochi University School of Medicine, Kohasu, Oko-cho, Nankoku, Kochi 783-8505, Japan

Received May 7, 2015; Accepted June 22, 2015

DOI: $10.3892 / o r .2015 .4171$

\begin{abstract}
The NOTCH family of membranous receptors plays key roles during development and carcinogenesis. Since NOTCH2, yet not NOTCH1 has been shown essential for murine hepatogenesis, NOTCH2 rather than NOTCH1 may be more relevant to human hepatocarcinogenesis; however, no previous studies have supported this hypothesis. We therefore assessed the role of $\mathrm{NOTCH} 2$ in human hepatocellular carcinoma (HCC) by immunohistochemistry and cell culture. Immunohistochemically, $19 \%$ of primary HCCs showed nuclear staining for $\mathrm{NOTCH} 2$, indicating activated $\mathrm{NOTCH} 2$ signaling. NOTCH2-positive HCCs were on average in more advanced clinical stages, and exhibited more immature cellular morphology, i.e. higher nuclear-cytoplasmic ratios and nuclear densities. Such features were not evident in NOTCH1-positive HCCs. In human HCC cell lines, abundant NOTCH2 expression was associated with anaplasia, represented by loss of E-cadherin. When NOTCH2 signaling was stably downregulated in HLF cells, an anaplastic HCC cell line, the cells were attenuated in potential for in vitro invasiveness and migration, as well as in vivo tumorigenicity accompanied by histological maturation. Generally, inverse results were obtained for a differentiated HCC cell line, Huh7, manipulated to overexpress activated NOTCH2. These findings suggested that the NOTCH2 signaling may confer aggressive behavior and immature morphology in human HCC cells.
\end{abstract}

\section{Introduction}

The NOTCH signaling pathway has been shown to have dual roles in development and carcinogenesis in multiple organs (1). In mammals, four NOTCH family genes, NOTCH1-4 have been described, each of which encodes a transmembrane receptor comprised of intracellular and extracellular domains. When

Correspondence to: Professor Gang-Hong Lee, Department of Pathology, Kochi University School of Medicine, Kohasu, Oko-cho, Nankoku, Kochi 783-8505, Japan

E-mail: ghlee@kochi-u.ac.jp

Key words: hepatocellular carcinoma, NOTCH2 signaling, aggressiveness, morphology a NOTCH ligand, e.g., a JAG or a DLL family protein, binds to the receptor, the intracellular domain is cleaved by the $\gamma$-secretase and translocated into the nucleus as an activated transcription factor for NOTCH target genes, including $H E S$ genes (1).

The relationship between NOTCH signaling and human hepatocarcinogenesis is still controversial. Both negative $(2,3)$ and positive $(4,5)$ correlations have been proposed. However, most studies have evaluated the effects of NOTCH through activating NOTCH1 or downstream NOTCH effectors common for the NOTCH family. Since NOTCH2, not NOTCH1 is essential for normal hepatic development in mice (6), more specific studies on human NOTCH2 are needed to support the established notion that cancer cells mimic immature features of their fetal counterparts.

To our knowledge, there is only one study on NOTCH2 expression in human hepatocellular carcinomas (HCCs); in this study, no nuclear localization of $\mathrm{NOTCH} 2$ protein was observed by immunohistochemistry analysis in any of the examined tumors (7). In the present study, we sought to investigate NOTCH2 signaling in human HCCs using tissue microarrays and cell lines. Contradictory to the previous study (7), our data supported that NOTCH2 had important roles in terms of aggressiveness and morphologic transformation of HCC cells.

\section{Materials and methods}

Tissue microarray and immunohistochemistry. We used tissue microarrays for human primary and metastatic HCCs (SuperBioChips Laboratories, Seoul, Korea). Immunohistochemical staining was performed as previously described (8), using primary antibodies against human activated NOTCH2 (ab52302), activated NOTCH1 (ab8925) (both from Abcam, Cambridge, UK), $\alpha$-fetoprotein (AFP), cytokeratin 19 (CK19) (both from Dako, Glostrup, Denmark), and EpCAM (ab187270; Abcam). The anti-NOTCH antibodies have been shown to react only with the activated forms of the intracellular domain after cleavage by $\gamma$-secretase (1). Clinical staging or histopathological grading of differentiation of the primary HCCs were performed according to the American Joint Committee on Cancer (AJCC) Cancer Staging Manual (9), or the General Rules for the Clinical and Pathological Study of Primary Liver Cancer (10), respectively. For quantification of the nuclear/cytoplasmic (N/C) ratio and nuclear density, the 
Table I. List of genes analyzed by semi-quantitative RT-PCR.

\begin{tabular}{|c|c|c|c|}
\hline Gene & Primer sequence ( $5^{\prime}$ to $\left.3^{\prime}\right)$ & Product size (bp) & PCR cycle no. \\
\hline NOTCH2 & $\begin{array}{l}\text { AAGCAGAGTCCCAGTGCCTA } \\
\text { CAGGGGGCACTGACAGTAAT }\end{array}$ & 173 & 36 \\
\hline$J A G 1$ & $\begin{array}{l}\text { GACTCATCAGCCGTGTCTCA } \\
\text { TGGGGAACACTCACACTCAA }\end{array}$ & 190 & 26 \\
\hline$J A G 2$ & $\begin{array}{l}\text { AGGTGGAGACGGTTGTTACG } \\
\text { TTGCACTGGTAGAGCACGTC }\end{array}$ & 250 & 41 \\
\hline$D L L 1$ & $\begin{array}{l}\text { TGTGCCTCAAGCAACTACCAG } \\
\text { TTCTGTTGCGAGGTCATCAG }\end{array}$ & 230 & 39 \\
\hline DLL3 & $\begin{array}{l}\text { GAGACACCCAGGTCCTTTGA } \\
\text { CAGTGGCAGATGTAGGCAGA }\end{array}$ & 152 & 38 \\
\hline$D L L 4$ & $\begin{array}{l}\text { TGCAGGAGTTCATCAACGAG } \\
\text { GAAATTGAAGGGCAGTTGGA }\end{array}$ & 227 & 38 \\
\hline HESI & $\begin{array}{l}\text { CGGACATTCTGGAAATGACA } \\
\text { CATTGATCTGGGTCATGCAG }\end{array}$ & 222 & 45 \\
\hline E-cadherin & $\begin{array}{l}\text { GCTGGAGATTAATCCGGACA } \\
\text { ACCTGAGGCTTTGGATTCCT }\end{array}$ & 237 & 38 \\
\hline$A L B$ & $\begin{array}{l}\text { TGCTTGAATGTGCTGATGACAGGG } \\
\text { AAGGCAAGTCAGCAGGCATCTCATC }\end{array}$ & 161 & 40 \\
\hline$G A P D H$ & $\begin{array}{l}\text { ACCACAGTCCATGCCATCAC } \\
\text { TCCACCACCCTGTTGCTGTA }\end{array}$ & 452 & 27 \\
\hline
\end{tabular}

$A L B$, albumin.

HCC tissues on the tissue microarray were photographed and analyzed using ImageJ software (http://rsb.info.nih.gov/ij/).

Cell lines and transfection. Six human HCC cell lines, Huh7, Hep3B, HepG2, HLE, HLF and PLC/5 were used for analyses. For transient knockdown of $\mathrm{NOTCH} 2$, we used anti-NOTCH2 siRNA (OriGene, Rockville, MD, USA). A total of $5 \times 10^{4}$ cells were inoculated into each well of a 6-well tissue culture plates and transfected with $5 \mu \mathrm{M}$ siRNA using the X-tremeGENE siRNA Transfection reagent (Roche, Basel, Switzerland). The cells were harvested $48 \mathrm{~h}$ after transfection, and protein and total RNA were collected for analysis. Universal scrambled negative control siRNA was used as a negative control (OriGene).

For stable knockdown of NOTCH2, the pRS plasmid vector harboring an shRNA targeting NOTCH2 and the puromycin-resistance gene were employed (OriGene). The same plasmid with a scrambled sequence was used as the negative control. For stable overexpression of $\mathrm{NOTCH} 2$, the pEF-BOS-neo SE plasmid vector with an expression construct of a partial NOTCH2 cDNA sequence for the intracellular region and the G418-resistance gene $(11,12)$ was obtained from Riken DNA Bank (Tsukuba, Japan). Stable transfectants were selected for 2 weeks with $2.0 \mu \mathrm{g} / \mathrm{ml}$ puromycin (Sigma, St. Louis, MO, USA) or $1 \mathrm{mg} / \mathrm{ml} \mathrm{G} 418$ (Roche) and clonal cell lines were established.

Western blot analysis. Monolayer cell cultures at $80 \%$ confluence were analyzed by western blotting as previously described (8), using primary antibodies against human activated NOTCH2 (ab72803; Abcam), E-cadherin (Dako) or $\beta$-actin (Sigma).

Semi-quantitative reverse transcription-polymerase chain reaction $(R T-P C R)$. Total RNA was isolated from cultured cells at $80 \%$ confluence using TRIzol reagent (Invitrogen, Carlsbad, CA, USA) and subsequent RT-PCR was performed as previously described (13). First-strand cDNAs were synthesized with oligo(dT) primer and the SuperScript III First-Strand Synthesis system (Invitrogen). Each single-stranded cDNA was diluted for subsequent PCR amplification. The standard PCR procedure was carried out in $15 \mu \mathrm{l}$ of PCR buffer. PCR conditions were: initial denaturation at $94^{\circ} \mathrm{C}$ for $7 \mathrm{~min}$, following by appropriate cycles of $94^{\circ} \mathrm{C}$ for $30 \mathrm{sec}, 55^{\circ} \mathrm{C}$ for $1 \mathrm{~min}$, and $72^{\circ} \mathrm{C}$ for $1 \mathrm{~min}$ and a final extension step of $72^{\circ} \mathrm{C}$ for $10 \mathrm{~min}$. Each PCR product $(15 \mu \mathrm{l})$ was run on native $7 \%$ polyacrylamide gels, stained with ethidium bromide and visualized by ultraviolet illumination. Staining intensity was quantified using ImageJ software. The screened gene panel included human NOTCH2, HES1, E-cadherin, albumin (ALB), JAG1, JAG2, DLL1, DLL3 and DLL4; GAPDH was screened as the control gene (Table I). The cycle number for each gene was determined as optimal when the amplification was within the linear range.

Transmembrane invasion and migration assays. In vitro invasion or migration assays were performed using BioCoat Matrigel invasion chambers in 24-well plates or those without 

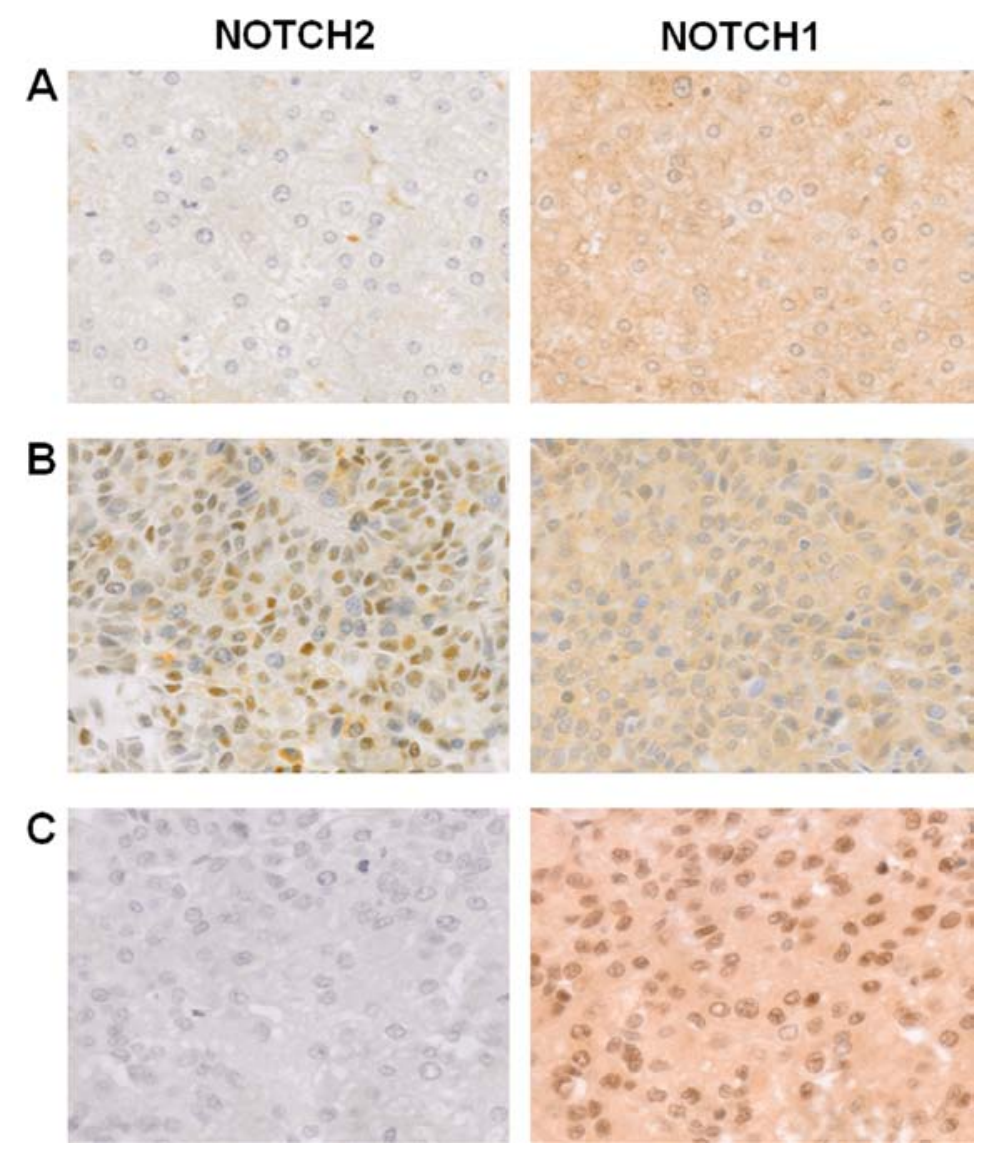

Figure 1. Immunohistochemical analysis of activated NOTCH2 and NOTCH1 using the liver tissue microarray. (A) A non-neoplastic liver tissue. (B) An HCC tissue positive for nuclear NOTCH2, but negative for nuclear NOTCH1. (C) An HCC tissue negative for nuclear NOTCH2, but positive for nuclear NOTCH1. Notably, the tumor in (B) is higher in N/C ratio and nuclear density than the tumor in (C). HCC, hepatocellular carcinoma; N/C, nuclear/cytoplasmic.

Matrigel (Becton-Dickinson, Franklin Lakes, NJ, USA), respectively (13). Suspensions of $2.5 \times 10^{4}$ cells in $0.5 \mathrm{ml}$ of serum-free Dulbecco's modified Eagle's medium (DMEM) were applied onto Matrigel-coated, $8-\mu \mathrm{m}$ pore-sized polycarbonate membranes at the bottom of each upper chamber, which was inserted into a lower well containing $0.75 \mathrm{ml}$ of DMEM with $10 \%$ fetal bovine serum as the chemoattractant. After $22 \mathrm{~h}$ of incubation, cells remaining on the upper surface of the membrane were removed with a cotton swab, and those that had invaded through to the bottom chamber were fixed with $4 \%$ paraformaldehyde and stained with crystal violet. Cells were observed under a light microscope, and four randomly selected fields were photographed at a magnification of $\mathrm{x} 100$. Invasion efficiency was expressed as the mean number of cells per photographed microscopic field. Each experiment was performed in quadruplicate. The protocol for the migration assay was the same as that for the invasion assay, except that a membrane without Matrigel coating was used (BioCoat control culture inserts; Becton-Dickinson).

In vivo tumorigenic assay. The animal experiments were approved by the Kochi University Animal Experiment Committee (permit no. G-00058). Immunodeficient male NOD. CB17-Prkdc $c^{\text {Scid } / J}$ (NOD SCID) mice (ages 8-10 weeks) were purchased from Charles River Laboratories Japan (Yokohama, Japan). Cultured cells were suspended in phosphate-buffered saline and $1 \times 10^{6}$ cells were transplanted into the subcutis of each mouse at the interscapular region. After 6 weeks, mice were sacrificed by cervical decapitation. Developed tumors were excised, weighed and fixed in $10 \%$ buffered formalin for histology.

Statistical analysis. For comparison of two independent mean values we employed the t-test. For the analysis of contingency tables, Fisher's exact test was applied. Differences were considered significant at P-values $<0.05$.

\section{Results}

Immunohistochemical analysis of NOTCH2 and NOTCH1. A total of 74 primary human HCCs and 9 non-neoplastic liver tissues, each adjacent to an independent primary HCC were immunohistochemically analyzed for activated intracellular domains of NOTCH2 and NOTCH1 (Fig. 1). While hepatocytes in the non-neoplastic livers showed weak cytoplasmic staining for both NOTCH2 and NOTCH1, the degree of staining for NOTCH2 was less intense under our reaction conditions (Fig. 1A). None of the non-neoplastic liver tissues exhibited a nuclear-predominant staining pattern for either NOTCH2 or NOTCH1 in hepatocytes. Since NOTCH signaling operates through nuclear localization of the activated intracellular domain (1), a tumor was judged positive only when $>10 \%$ of the cells exhibited distinct nuclear-predominant staining (Fig. 1B and C). This cut-off criterion was set 
Table II. Clinicopathological features of 74 primary HCCs based on nuclear-predominant immunohistochemical staining for NOTCH2 or NOTCH1.

\begin{tabular}{|c|c|c|c|c|c|c|}
\hline & \multicolumn{3}{|c|}{ NOTCH2 } & \multicolumn{3}{|c|}{ NOTCH1 } \\
\hline & $\begin{array}{l}\text { Positive } \\
\text { cases }^{\mathrm{a}}\end{array}$ & $\begin{array}{l}\text { Negative } \\
\text { cases }^{\text {b }}\end{array}$ & P-value & $\begin{array}{l}\text { Positive } \\
\text { cases }^{\mathrm{a}}\end{array}$ & $\begin{array}{l}\text { Negative } \\
\text { cases }^{\text {b }}\end{array}$ & P-value \\
\hline $\begin{array}{l}\text { Analyzed cases } \\
74 \text { primary HCCs }\end{array}$ & $14(19 \%)$ & $60(81 \%)$ & NA & $18(24 \%)$ & $56(76 \%)$ & NA \\
\hline Age, mean (years) & 56.7 & 55.0 & NS & 54.1 & 55.7 & NS \\
\hline \multicolumn{7}{|l|}{ Gender } \\
\hline Male/female & $14 / 0$ & $44 / 16$ & 0.031 & $15 / 3$ & $43 / 13$ & NS \\
\hline \multicolumn{7}{|l|}{ Clinical stage $(\text { score })^{\mathrm{c}}$} \\
\hline I (1)/II (2)/III (3)/IV (4) & $1 / 4 / 7 / 2$ & $23 / 17 / 18 / 2$ & & $3 / 6 / 8 / 1$ & $21 / 15 / 17 / 3$ & \\
\hline Mean score & 2.71 & 1.98 & 0.004 & 2.39 & 2.04 & NS \\
\hline \multicolumn{7}{|l|}{ Histological grade (score) ${ }^{\mathrm{d}}$} \\
\hline Mean score & 2.00 & 1.93 & NS & 1.92 & 1.95 & NS \\
\hline
\end{tabular}

NA, not applicable; NS, not significant; well, well differentiated; mod, moderately differentiated; poor, poorly differentiated. ${ }^{\mathrm{a}} \mathrm{More}$ than $10 \%$ of tumor cells show predominantly nuclear staining. ${ }^{\text {Less }}$ than or equal to $10 \%$ of tumor cells show predominantly nuclear staining. ${ }^{\mathrm{C}}$ The AJCC Cancer Staging Manual, 7th edition (9). ${ }^{\mathrm{d}}$ The Clinical and Pathological Study of Primary Liver Cancer, 8th edition (10). HCC, hepatocellular carcinoma.

Table III. Comparison of independent sets of primary and metastatic HCCs analyzed for nuclear NOTCH2 and NOTCH1 using immunohistochemistry.

\begin{tabular}{|c|c|c|c|c|c|c|c|}
\hline \multirow[b]{2}{*}{$\mathrm{HCC}$} & \multirow[b]{2}{*}{$\begin{array}{l}\text { Total } \\
\text { cases }\end{array}$} & \multicolumn{3}{|c|}{ NOTCH2 } & \multicolumn{3}{|c|}{ NOTCH1 } \\
\hline & & $\begin{array}{c}\text { Positive } \\
\operatorname{cases}^{\mathrm{a}}(\%)\end{array}$ & $\begin{array}{c}\text { Negative } \\
\text { cases }^{\mathrm{b}}(\%)\end{array}$ & P-value & $\begin{array}{c}\text { Positive } \\
\operatorname{cases}^{\mathrm{a}}(\%)\end{array}$ & $\begin{array}{c}\text { Negative } \\
\operatorname{cases}^{\mathrm{b}}(\%)\end{array}$ & P-value \\
\hline Primary & 74 & 14 (19) & $60(81)$ & & $18(24)$ & $56(76)$ & \\
\hline Metastatic & 18 & $11(61)$ & 7 (39) & 0.001 & $12(67)$ & $6(33)$ & 0.001 \\
\hline
\end{tabular}

according to a preliminary test for reproducibility of the judgment between two observers. On average, NOTCH2-positive tumors were in significantly more advanced clinical stages than NOTCH2-negative tumors (Table II). A similar trend was observed for NOTCH1-positive tumors, although this result did not reach statistical significance. In addition, all NOTCH2-positive cases were observed in men, and this difference was statistically significant. There was no significant correlation between the histopathological grade of differentiation and positive staining for either NOTCH2 or NOTCH1.

We next analyzed metastatic HCC tissues in various organs obtained from an independent set of 18 patients. The NOTCH2-positive rate was significantly higher in metastatic HCCs, and the same conclusion was reached with NOTCH1 (Table III). In primary HCCs, NOTCH2-positive HCCs showed no significant difference in NOTCH1 positivity compared with the NOTCH2-negative HCCs. However, in metastatic $\mathrm{HCCs},>90 \%$ of NOTCH2-positive tumors were simultaneously positive for NOTCH1 (Table IV).

During the immunohistochemical analysis, we noticed that cells in NOTCH2-positive HCCs tended to be small in size and high in N/C ratio (Fig. 1), reminiscent of immature hepatoblasts. Thus, we quantified N/C ratios and nuclear densities in primary and metastatic HCC cases (Fig. 2). Among the 25 NOTCH2-positive HCCs on the tissue microarray, three cases were excluded from the analysis due to their low tumor cell content on the array. As controls, $23 \mathrm{NOTCH} 2$-negative HCCs were randomly selected and simultaneously analyzed, and both of the parameters were significantly higher for NOTCH2-positive HCCs compared with NOTCH2-negative HCCs. Such features were not evident for NOTCH1-positive HCCs. Furthermore, tumors positive for only NOTCH2 showed significantly higher mean $\mathrm{N} / \mathrm{C}$ ratios and nuclear densities than those positive for only NOTCH1. 
Table IV. Correlation between immunohistochemical staining for nuclear NOTCH2 and NOTCH1 in primary or metastatic HCCs.

\section{NOTCH1}

\begin{tabular}{|c|c|c|c|c|c|c|}
\hline & \multicolumn{3}{|c|}{ Primary HCC cases } & \multicolumn{3}{|c|}{ Metastatic HCC cases } \\
\hline & Positive $^{\mathrm{a}}(\%)$ & Negative $^{\mathrm{b}}(\%)$ & P-value & Positive $^{a}(\%)$ & Negative $^{\mathrm{b}}(\%)$ & P-value \\
\hline \multicolumn{7}{|l|}{ NOTCH2 } \\
\hline Positive $^{\mathrm{a}}$ & $2(14)$ & $12(86)$ & & $10(91)$ & $1(9)$ & \\
\hline Negative $^{\mathrm{b}}$ & $16(27)$ & $44(73)$ & NS & $2(29)$ & $5(71)$ & 0.013 \\
\hline
\end{tabular}

NS, not significant. ${ }^{a}$ More than $10 \%$ of tumor cells show predominantly nuclear staining. ${ }^{\text {bess }}$ than or equal to $10 \%$ of tumor cells show predominantly nuclear staining. HCCs, hepatocellular carcinomas.

A

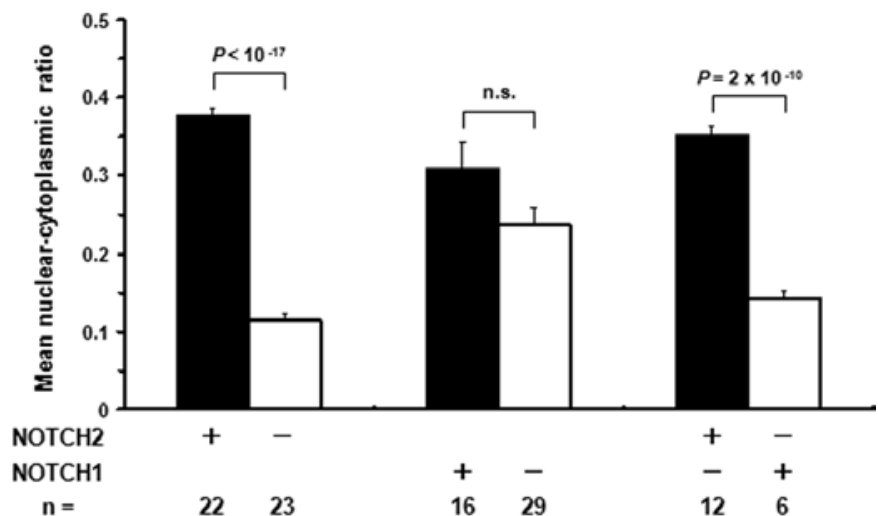

B

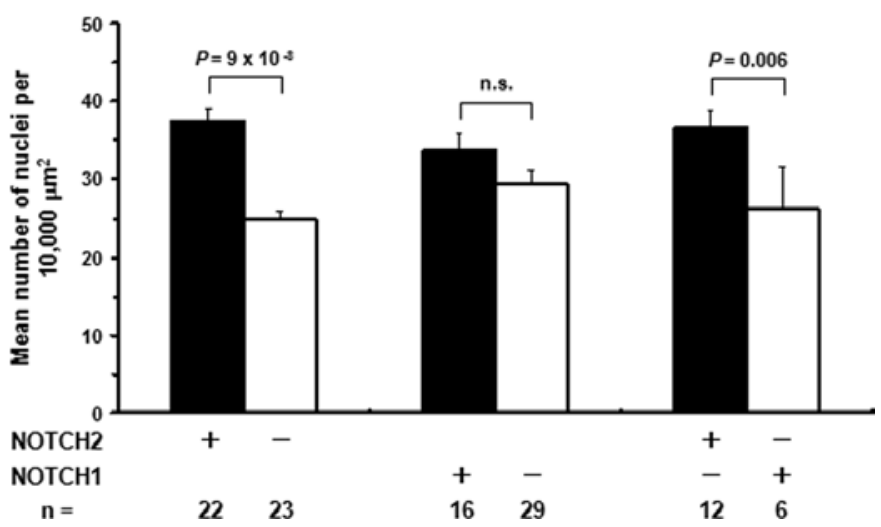

Figure 2. Quantification of the nuclear-cytoplasmic ratio and nuclear density. (A) Mean nuclear-cytoplasmic ratio and (B) mean nuclear density of 34 primary and 11 metastatic hepatocellular carcinomas randomly selected from the tissue microarray in relation to their immunohistochemical staining for nuclear NOTCH2 or NOTCH1.

Expression of hepatoblast markers in NOTCH2-positive HCCs. Since the NOTCH2 signaling was associated with immaturemorphology of HCCcells, we immunohistochemically analyzed expression of 3 representative hepatoblast markers, AFP, CK19 and EpCAM (14), with 14 NOTCH2-positive and 44 NOTCH2-negative primary HCCs (Table V). A tumor was judged positive when $>10 \%$ of the cells exhibited distinct staining in order to keep the reproducibility of the judgment between two observers. Compared with the NOTCH2-negative HCCs, the NOTCH2-positive HCCs were more frequently positive for AFP and also larger in the mean multiplicity of positive hepatoblast markers per case.
NOTCH2 expression in human HCC cell lines. To determine whether NOTCH2 actually controlled aggressive behavior and immature cellular morphology of HCC cells, we examined NOTCH2 expression in six human HCC cell lines by western blot analysis (Fig. 3). Anti-NOTCH2 antibodies specifically recognizing the activated NOTCH2 intracellular domain demonstrated that this domain was detectable in all cell lines analyzed. However, the expression levels varied. For example, two HCC cell lines, HLE and HLF, expressed relatively high levels of NOTCH2, accompanied by loss of E-cadherin expression as a marker protein for differentiated epithelium. The other cell lines tested were positive for E-cadherin and 
Table V. Expression of representative hepatoblast markers, AFP, CK19 and EpCAM, in NOTCH2-positive and -negative primary HCCs.

\begin{tabular}{lccccc}
\hline & & \multicolumn{3}{c}{ Positive cases $^{\mathrm{a}}$} & $\begin{array}{r}\text { Mean multiplicity of } \\
\text { positive markers/case }\end{array}$ \\
\cline { 3 - 4 } & Total cases & AFP & EpCAM & CK19 & \\
\hline NOTCH2 & & & & & 1.43 \\
Positive & 14 & $7(50 \%)$ & $10(71 \%)$ & $3(21 \%)$ & 0.75 \\
Negative & 44 & $9(20 \%)$ & $19(43 \%)$ & $5(11 \%)$ & 0.024 \\
P-value & & 0.043 & NS & NS & \\
\hline
\end{tabular}

NS, not significant. ${ }^{a}$ More than $10 \%$ of tumor cells show staining. AFP, $\alpha$-fetoprotein; HCCs, hepatocellular carcinomas.

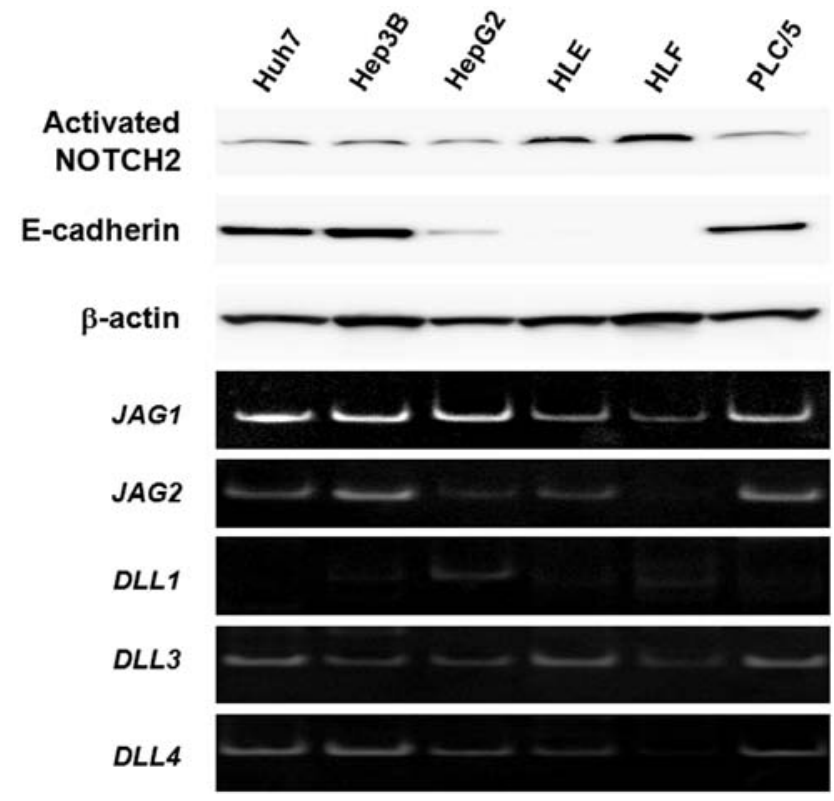

Figure 3. Characterization of human hepatocellular carcinoma cell lines. Western blot analysis of activated NOTCH2, E-cadherin and $\beta$-actin proteins, and RT-PCR analysis of mRNAs for NOTCH ligand genes, i.e., JAG1, $J A G 2, D L L 1, D L L 3$ and $D L L 4$.

exhibited relatively lower NOTCH2 expression. Consequently, there was an apparent correlation between cellular anaplasia and NOTCH 2 activation in cultured HCC cells. RT-PCR analyses of NOTCH ligand genes, i.e., JAG1, JAG2, DLL1, DLL3 and $D L L 4$ (Fig. 3), indicated that each cell line expressed all of these genes; however, the expression levels of these targets varied between the cell lines.

Knockdown of NOTCH2 expression in HLF cells. To further clarify the role of NOTCH2 signaling, we used the HLF cell line with a high NOTCH2 expression, for gene knockdown experiments. We first transiently transfected the cells with siRNA targeting NOTCH2 (Fig. 4). HLF cells were revealed to be anaplastic HCC cells that do not express detectable amounts of mRNA for E-cadherin or ALB, a mature hepatocyte marker. However, $48 \mathrm{~h}$ after transfection, expression of $E$-cadherin and $A L B$, mRNAs was induced, concurrent with the reductions in $\mathrm{mRNA}$ /protein for NOTCH2 and mRNA for $H E S 1$, a representative NOTCH target gene (1).

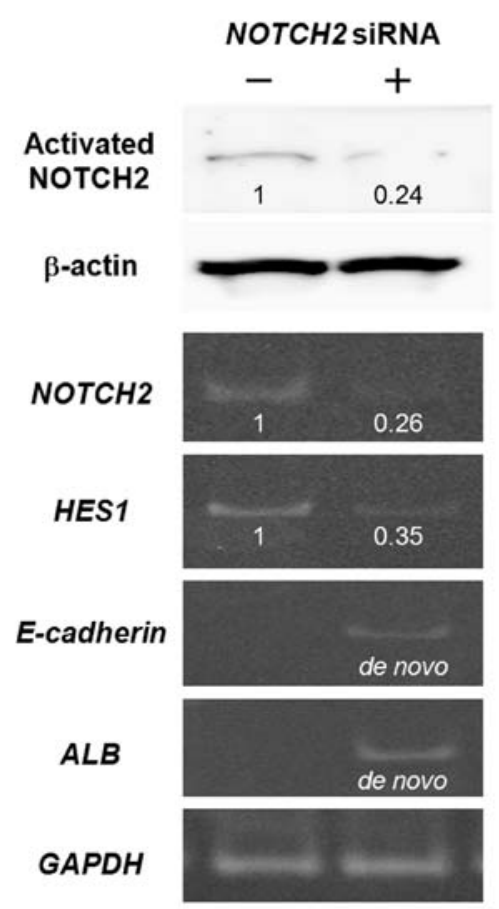

Figure 4. Effects of transient knockdown of NOTCH2 expression in HLF cells. Forty-eight hours after transfection of HLF cells with negative control RNA or NOTCH2 siRNA, western blot analysis of activated NOTCH2 and $\beta$-actin proteins, and RT-PCR analysis of mRNAs for NOTCH2, HES1, $E$-cadherin, $A L B$ and GAPDH genes were performed. Each numerical value is the relative expression level normalized to the level of $\beta$-actin protein or GAPDH mRNA. E-cadherin and ALB mRNAs were induced de novo after the siRNA transfection. $A L B$, albumin.

We then attempted to generate an HLF clone with reduced levels of $\mathrm{NOTCH} 2$ by stable transfection of NOTCH2 shRNA. Western blotting revealed that NOTCH2 expression was most effectively inhibited in a clone, termed HLF-kd, which was further characterized in comparison with a clone mock-transfected with vector only, HLF-mock (Fig. 5A). HLF-kd cells showed de novo expression of E-cadherin. The NOTCH target gene $H E S I$ exhibited decreased mRNA expression, confirming that $\mathrm{NOTCH} 2$ signaling was inhibited in HLF-kd cells. Morphologically, while HLF-mock cells as well as parental HLF cells were spindle in shape, HLF-kd cells were polygonal and more epithelial-like (Fig. 5B). We next evaluated the effects of NOTCH2 knockdown on migration and invasion 
A
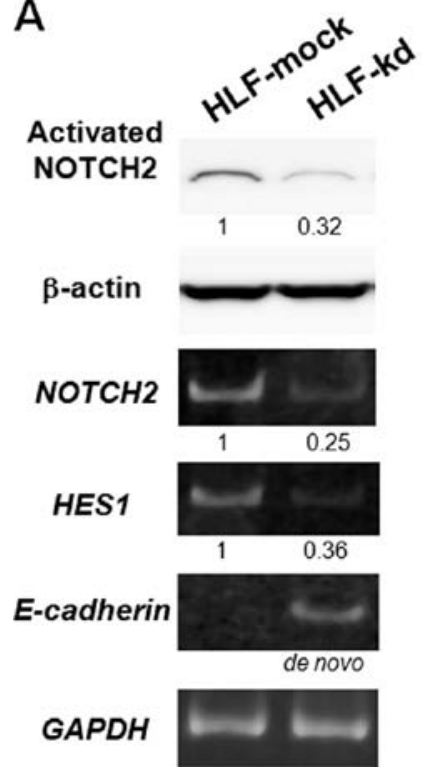
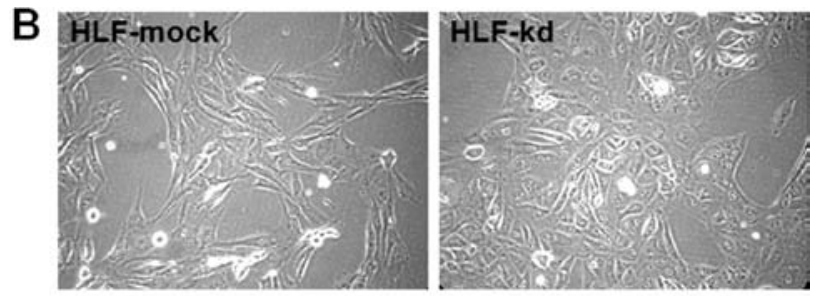

C

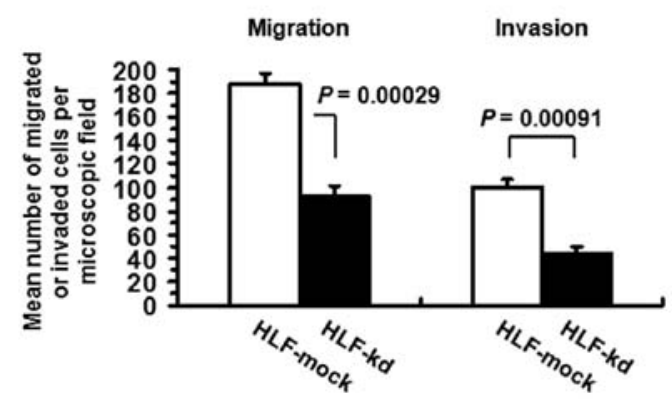

Figure 5. Characterization of HLF cells with stable knockdown of NOTCH2. HLF-kd is a clonal cell line stably transfected with NOTCH2 shRNA. HLF-mock is a mock transfected clone that served as a negative control. (A) Western blot analysis of activated NOTCH2 and $\beta$-actin proteins, and RT-PCR analysis of mRNAs for NOTCH2, HES1, E-cadherin and GAPDH genes were performed. Each numerical value is the relative expression level normalized to the level of $\beta$-actin protein or GAPDH mRNA. E-cadherin mRNA was induced de novo in HLF-kd cells. (B) Phase-contrast photomicrographs of HLF-mock and HLF-kd cells. (C) Results of in vitro migration and invasion assays with HLF-mock and HLF-kd cells. Each experiment was performed in quadruplicate and mean values are shown. Bars indicate standard errors.
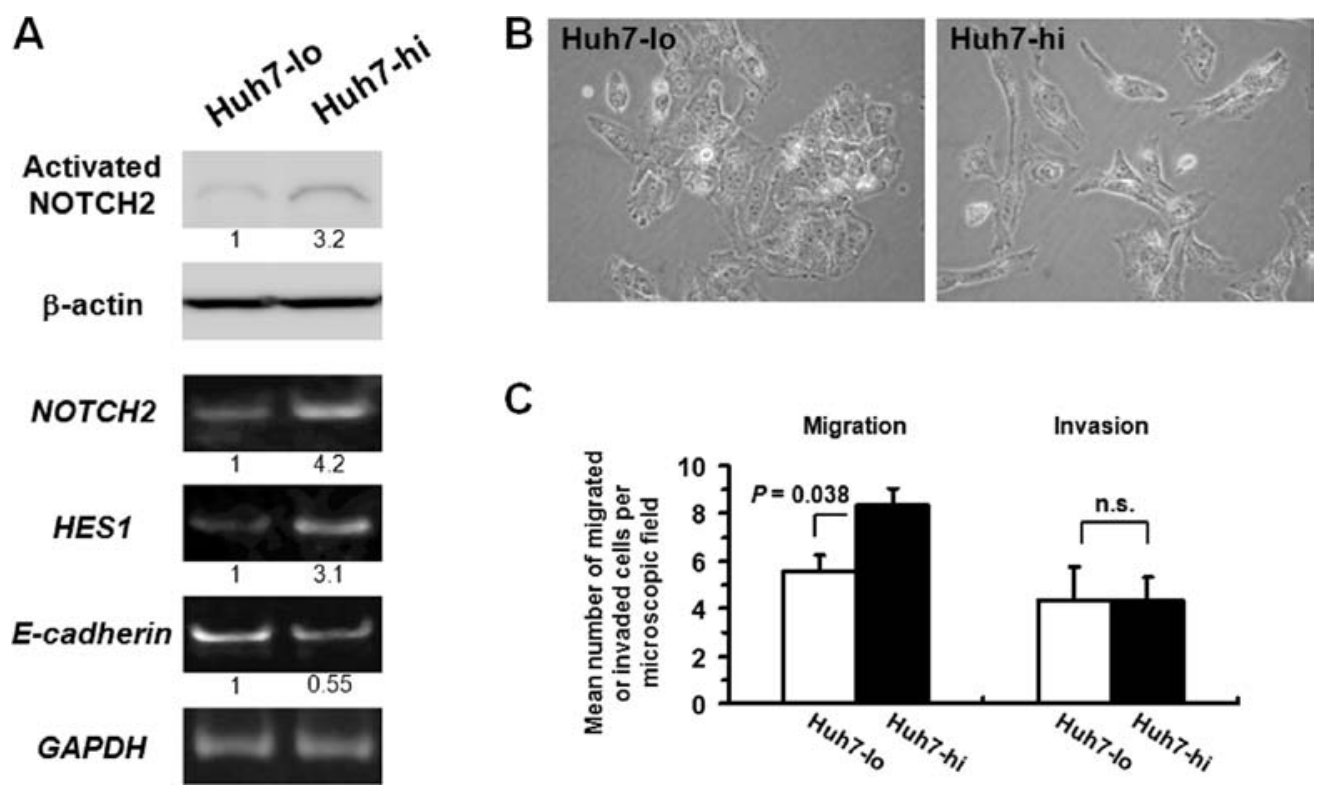

C

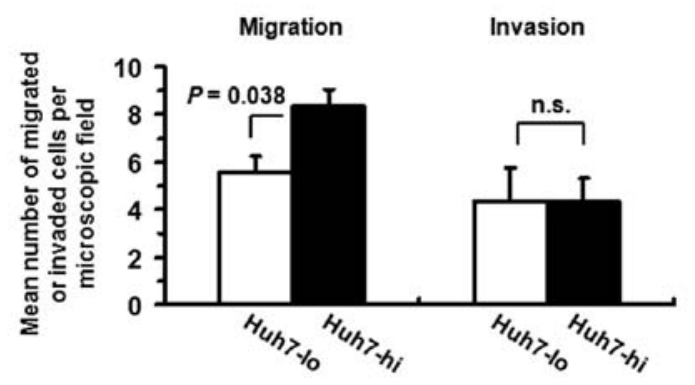

Figure 6. Characterization of Huh7 cells stably transfected with a gene for the NOTCH2 intracellular domain. Clonal cell lines were established after stable transfection of Huh7 cells with a plasmid construct for overexpression of the NOTCH2 intracellular domain. Among the cell lines, Huh7-hi exhibited the highest levels of activated NOTCH2. The level of activated NOTCH2 in Huh7-lo cells was equivalent to that in the parental Huh7 cell line. (A) Western blot analysis of activated NOTCH2 and $\beta$-actin proteins and RT-PCR analysis of mRNAs for NOTCH2, HES1, E-cadherin and GAPDH genes were performed. Each numerical value is the relative expression level normalized to the level of $\beta$-actin protein or GAPDH mRNA. (B) Phase-contrast photomicrographs of Huh7-lo and Huh7-hi cells. (C) Results of in vitro migration and invasion assays with Huh7-lo and Huh7-hi cells. Each experiment was performed in quadruplicate and mean values are shown. Bars indicate standard errors.

in HLF cells in vitro (Fig. 5C). HLF-kd cells exhibited significantly reduced migratory potential and invasiveness compared with control HLF-mock cells.

Overexpression of NOTCH2 intracellular domain in Huh7 cells. Among the six HCC cell lines tested, three, including Huh7, retained high E-cadherin expression with relatively low
NOTCH2 expression levels (Fig. 3). In order to clarify the effects of induced overexpression of $\mathrm{NOTCH} 2$, we transfected Huh7 cells with an expression construct for the NOTCH2 intracellular domain. One clone with the highest expression of NOTCH2 was isolated (Huh7-hi), as was the clone with the lowest NOTCH2 expression (Huh7-lo; Fig. 6A). Compared with Huh7-lo cells, Huh7-hi cells exhibited an increase in 
A
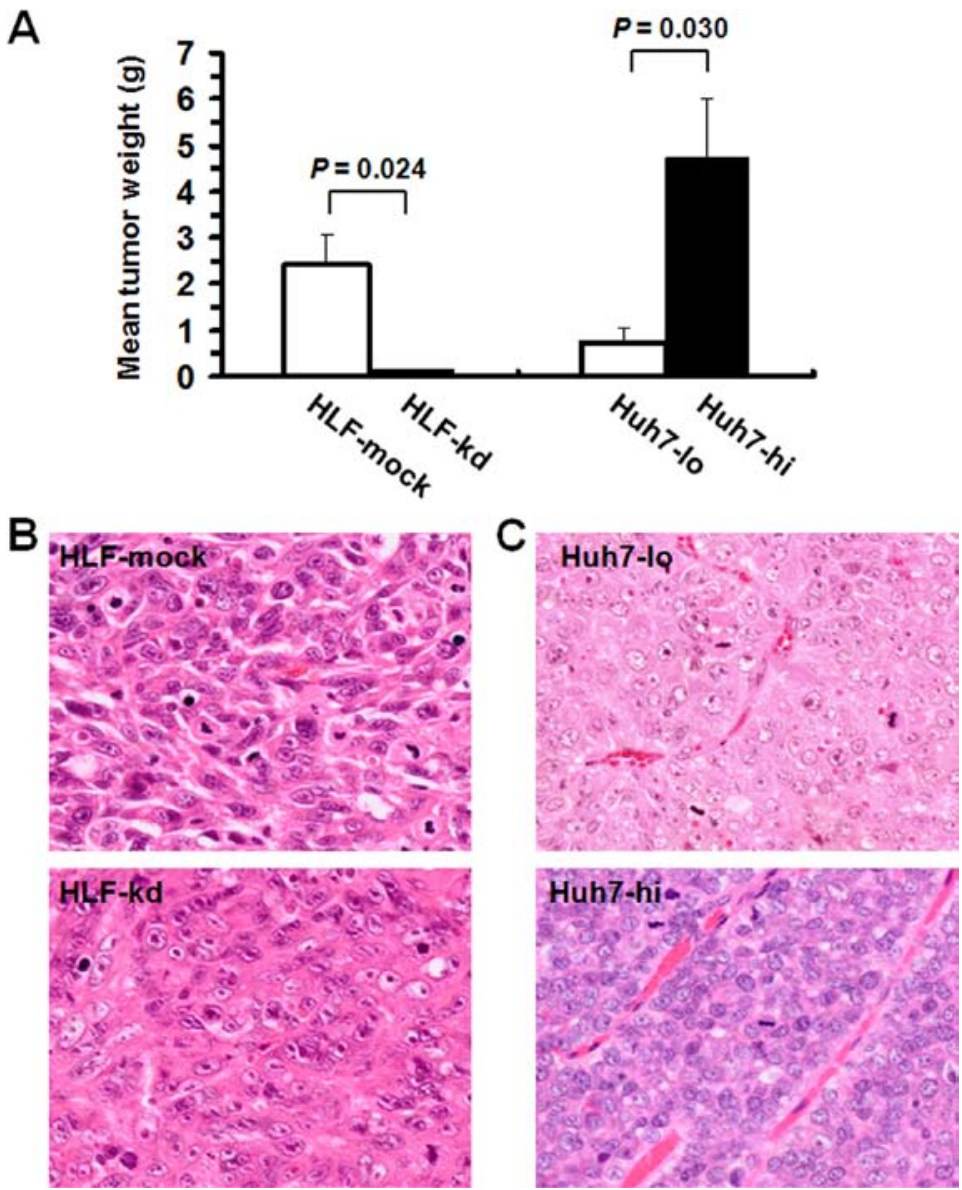

Figure 7. In vivo tumorigenicity assay using immunodeficient mice. (A) Mean weights of transplanted tumors. Five mice were used for each cell line. Bars indicate standard errors. (B and C) Histologies of HLF-mock and HLF-kd cells (B) or Huh7-lo and Huh7-hi cells (C) transplanted into the mouse subcutis.

HES1 mRNA and a modest decrease in E-cadherin mRNA. Morphologically, while Huh7-lo cells were typically epitheliallike, represented by their polygonal shape and tight adhesion, Huh7-hi cells were more elongated and scattered (Fig. 6B). In vitro migration and invasion assays revealed that Huh7-hi cells acquired a modest increase in migratory potential, but no apparent enhancement in invasiveness compared with Huh7-lo cells (Fig. 6C). Both Huh7-hi and -lo cells exhibited only $<5 \%$ of the migratory or invasive activity relative to HLF-mock cells, indicating that the intrinsic migratory and invasive potentials of Huh7 cells were limited.

Effects of NOTCH2 expression on in vivo tumorigenicity and morphology of HCC cell lines in vivo. The results of in vitro experiments indicated that $\mathrm{NOTCH} 2$ signaling causes immature morphology and biological aggressiveness in human HCC cells. We thus performed in vivo tumorigenicity assays using immunodeficient NOD SCID mice and HCC cells with stable modifications to NOTCH2 expression. HLF-kd-based tumors exhibited significantly lower mean tumor weights than control HLF-mock-based tumors (Fig. 7A). Histologically, tumors arising from HLF-mock cells were highly anaplastic, with hyperchromatic nuclei, and exhibited increased cellularity and N/C ratios (Fig. 7B). On the contrary, tumors arising from HLF-kd cells exhibited more hepatocyte-like cellular morphologies, with relatively abundant, eosinophilic cytoplasms and vesicular nuclei with prominent nucleoli.
The same experiments were performed with Huh7-hi and Huh7-lo cells. Huh7-hi-based tumors exhibited significantly higher mean tumor weights than tumors arising from Huh7-lo cells (Fig. 7A). Tumors arising from Huh7-hi or Huh7-lo cells were histopathologically diagnosed as moderately to poorly differentiated, trabecular HCCs (Fig. 7C). However, relative to the tumors arising from Huh7-lo cells, those arising from Huh7-hi cells exhibited a more immature morphology characterized by high cellularity and N/C ratio.

\section{Discussion}

Our results indicated that, contradictory to the results of a previous study (7), activation of NOTCH2 signaling is not a rare event in clinically advanced or metastatic HCCs. We further provided evidence that NOTCH2 signaling induces morphological immaturity in HCC cells, while NOTCH1 activation did not seem to be tightly linked to this phenotype. The immature morphology of individual HCC cells was not necessarily associated with poor grade of differentiation, since NOTCH2 signaling did not appear to affect the structural atypism.

Unlike HCCs, most human hepatoblastomas have been shown to be positive for nuclear NOTCH2 using immunohistochemistry analysis (15). Hepatoblastoma cells, particularly of embryonal type, are morphologically immature, exhibiting small sizes and high N/C ratios. Thus, NOTCH2-positive 
HCC cells share some morphological characteristics with hepatoblastoma cells. In fact, NOTCH2-positive HCCs more frequently expressed representative hepatoblast markers, AFP, CK19 and EpCAM, than NOTCH2-negative HCCs. However, in contrast to typical hepatoblastomas, half of the NOTCH2-positive HCCs were immunohistochemically negative for AFP, implying that NOTCH2-positive HCC cells may not be simply regarded as equivalent to hepatoblastoma cells.

In mouse models, constitutive activation of either NOTCH1 or NOTCH2 specifically in the liver has been shown to promote hepatocellular tumor development $(5,16)$. In addition, recent studies using cultured human HCC cells revealed that NOTCH1 activation augments the migration and invasiveness of HCC cells in vitro $(17,18)$. These observations, along with our present results, indicate that both NOTCH1 and NOTCH2 may be capable of promoting human hepatocarcinogenesis. In particular, our data on primary and metastatic $\mathrm{HCCs}$ suggested that cooperation between NOTCH2 and NOTCH1 signaling may confer a selective advantage for metastasis in HCC cells. However, we found that only NOTCH2 was significantly associated with advanced clinical staging in primary HCCs. Moreover, some earlier studies proposed that NOTCH1 signaling may inhibit human hepatocarcinogenesis through induction of apoptosis in HCC cells $(6,7)$. Therefore, NOTCH2 rather than NOTCH1 may be more important for the promotion of hepatocarcinogenesis.

The correlation between activated NOTCH 2 signaling and advanced clinical stage or metastasis suggested the potential usefulness of NOTCH2 as a predictor of prognosis in patients with HCC. This idea was also supported by our in vitro migration and invasion assay results or the results of our in vivo tumorigenicity assay using human HCC cell lines. Notably, all NOTCH2-positive primary HCC patients in the present study were men, which probably reflected the poorer prognosis of male HCC patients than female HCC patients (19).

\section{Acknowledgements}

We thank Dr Tasuku Honjo and Dr Shigekazu Nagata for their distribution of the expression vector plasmid for the NOTCH2 intracellular domain through Riken DNA Bank. This study was supported in part by grant from the Grants-in-Aid for Scientific Research from the Japan Society for the Promotion of Science.

\section{References}

1. Tien AC, Rajan A and Bellen HJ: A Notch updated. J Cell Biol 184: 621-629, 2009.

2. Kureishy N, Sapountzi V, Prag S, Anilkumar N and Adams JC: Fascins, and their roles in cell structure and function. BioEssays 24: 350-361, 2002.
3. Wang C, Qi R, Li N, Wang Z, An H, Zhang Q, Yu Y and Cao X: Notch1 signaling sensitizes tumor necrosis factor-related apoptosis-inducing ligand-induced apoptosis in human hepatocellular carcinoma cells by inhibiting Akt/Hdm2-mediated p53 degradation and up-regulating p53-dependent DR5 expression. J Biol Chem 284: 16183-16190, 2009.

4. Wang XQ, Zhang W, Lui EL, Zhu Y, Lu P, Yu X, Sun J, Yang S, Poon RT and Fan ST: Notch1-Snaill-E-cadherin pathway in metastatic hepatocellular carcinoma. Int $\mathbf{J}$ Cancer 131: E163-E172, 2012.

5. Villanueva A, Alsinet C, Yanger K, Hoshida Y,Zong Y, Toffanin S, Rodriguez-Carunchio L, Solé M, Thung S, Stanger BZ, et al: Notch signaling is activated in human hepatocellular carcinoma and induces tumor formation in mice. Gastroenterology 143: 1660-1669.e7, 2012.

6. Geisler F, Nagl F, Mazur PK, Lee M, Zimber-Strobl U, Strobl LJ, Radtke F, Schmid RM and Siveke JT: Liver-specific inactivation of Notch2, but not Notch1, compromises intrahepatic bile duct development in mice. Hepatology 48: 607-616, 2008.

7. Gao J, Song Z, Chen Y, Xia L, Wang J, Fan R, Du R, Zhang F, Hong L, Song J, et al: Deregulated expression of Notch receptors in human hepatocellular carcinoma. Dig Liver Dis 40: 114-121, 2008.

8. Yamasaki K, Hayashi Y, Okamoto S, Osanai M and Lee GH: Insulin-independent promotion of chemically induced hepatocellular tumor development in genetically diabetic mice. Cancer Sci 101: 65-72, 2010.

9. Edge S, Byrd DR, Compton CC, Fritz AG, Greene FL and Trotti A (eds): AJCC Cancer Staging Manual. 7th edition. Springer, New York, 2010.

10. Liver Cancer Study Group of Japan (ed): The Clinical and Pathological Study of Primary Liver Cancer, 5th edition. Kanehara Press, Tokyo, 2008.

11. Mizushima S and Nagata S: pEF-BOS, a powerful mammalian expression vector. Nucleic Acids Res 18: 5322, 1990.

12. Kato H, Sakai T, Tamura K, Minoguchi S, Shirayoshi Y, Hamada Y, Tsujimoto Y and Honjo T: Functional conservation of mouse Notch receptor family members. FEBS Lett 395: 221-224, 1996.

13. Hayashi Y, Osanai M and Lee GH: Fascin-1 expression correlates with repression of E-cadherin expression in hepatocellular carcinoma cells and augments their invasiveness in combination with matrix metalloproteinases. Cancer Sci 102: 1228-1235, 2011.

14. Zhang L, Theise N, Chua M and Reid LM: The stem cell niche of human livers: Symmetry between development and regeneration. Hepatology 48: 1598-1607, 2008.

15. Litten JB, Chen TT, Schultz R, Herman K, Comstock J, Schiffman J, Tomlinson GE and Rakheja D: Activated NOTCH2 is overexpressed in hepatoblastomas: An immunohistochemical study. Pediatr Dev Pathol 14: 378-383, 2011.

16. Dill MT, Tornillo L, Fritzius T, Terracciano L, Semela D, Bettler B, Heim MH and Tchorz JS: Constitutive Notch2 signaling induces hepatic tumors in mice. Hepatology 57: 1607-1619, 2013.

17. Zhou L, Wang DS, Li QJ, Sun W, Zhang Y and Dou KF: The down-regulation of Notch1 inhibits the invasion and migration of hepatocellular carcinoma cells by inactivating the cyclooxygenase-2/Snail/E-cadherin pathway in vitro. Dig Dis Sci 58: 1016-1025, 2013

18. Zhou L, Zhang N, Song W, You N, Li Q, Sun W, Zhang Y, Wang D and Dou K: The significance of Notch1 compared with Notch3 in high metastasis and poor overall survival in hepatocellular carcinoma. PLoS One 8: e57382, 2013.

19. Ng IO, Ng MM, Lai EC and Fan ST: Better survival in female patients with hepatocellular carcinoma. Possible causes from a pathologic approach. Cancer 75: 18-22, 1995. 